\title{
Pull test performance and correlation with falls risk in Parkinson's disease
}

\section{Performance e correlação do teste de retropulsão com o risco de quedas na doença de Parkinson}

Renato P. Munhoz ${ }^{1,2}$, Helio A. Teive

\begin{abstract}
Postural instability (PI) and falls are major sources of disability in Parkinson's disease (PD). Our objectives were to evaluate the correlation between the pull-test (PT) scores and falls. Patients underwent a standardized data collection including demographic, clinical data, and the UPDRS scores for falls and the PT. Cases with scores $>1$ for falls were considered frequent fallers. 264 patients were included with mean age $67.6 \pm 10$ years, mean age of onset $59.1 \pm 10.7$ years. Comparison between PT scores versus the proportion of frequent fallers and the mean score of the UPDRS for falls showed that for each increase in the PT score, both parameters were significantly worse, with positive linear relationship. For any abnormal PT score, sensibility and negative predictive value were excellent; specificity and positive predictive value improved with worse PT scores. In conclusion, the PT provides important and reliable information regarding PI and the risk of falls in PD.
\end{abstract}

Keywords: Parkinson's disease, pull test, postural instability, falls.

\section{RESUMO}

Instabilidade postural (IP) e quedas são importantes fontes de incapacidade na doença de Parkinson (DP). Nossos objetivos foram avaliar a correlação dos escores do test de retropulsão (TR) com história de quedas. Pacientes foram submetidos a coleta de dados padronizada, incluindo dados demograficos, clinicos e escores da UPDRS para quedas e do TR. Escores $>1$ para quedas definiram casos com quedas

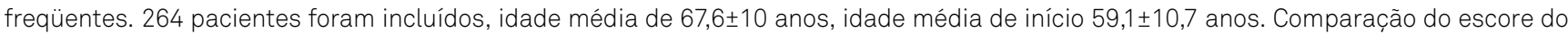
TR com a proporção de casos com quedas freqüentes e pontuação média para quedas mostrou que, para cada aumento na pontuação do TR, ambos os parâmetros pioraram significativamente, com relação linear. Para escores anormais no TR, sensibilidade e valor preditivo negativo foram excelentes; especificidade e valor preditivo positivo melhoraram com escores maiores. Conclusão: o TR fornece informações importantes e confiáveis sobre IP e risco de quedas em PD.

Palavras-chave: doença de Parkinson, teste de retropulsão, instabilidade postural e quedas.

Although postural instability (PI) and falls are rare as presenting features in patients with Parkinson's disease (PD), they represent major sources of disability and limitation in quality of life measures as the disease progresses and overall disability increases ${ }^{1}$. PI is also a critical milestone in the course of the disease, a landmark of disease progression as it defines the Hoehn and Yahr disability scale (H\&Y) stage $\mathrm{III}^{2}$. Typically, after reaching stage III the disease course enters more challenging phases, classified as moderate to severe, marked by more debilitating motor and non-motor symptoms and signs that are resistant to pharmacological and surgical interventions, including bulbar signs, gait and cognitive disturbances ${ }^{3}$. Although falls are considered the most dangerous complication of PI and gait disorders, other consequences, such as fear of falling and the effort to keep a safe gait pattern, are also sources of functional impairment ${ }^{4}$.

Several tests have been used to identify individuals with PI, such as the pull-test (PT), the push-release test, tandem stance and gait, single-leg standing, and the timed up-and-go tests. Additionally, quantitative posturography can also provide objective measures of $\mathrm{PI}^{5,6}$.

Although the utility and technical performance of the PT have been questioned ${ }^{6}$, it remains by far the most widely known of such tests, mainly because its' performance and

${ }^{1}$ Movement Disorders Centre, Toronto Western Hospital, University of Toronto, Toronto ON, Canada;

${ }^{2}$ Associação Paranaense dos Portadores de Parkinsonismo, Curitiba PR, Brazil;

${ }^{3}$ Unidade de Desordens do Movimento, Serviço de Neurologia, Hospital de Clínicas, Universidade Federal do Paraná, Curitiba PR, Brazil.

Correspondence: Renato P. Munhoz; 399 Bathurst Street, ML-7; Toronto ON, Canada, M5T 2S8; E-mail: renato.munhoz@uhn.ca

Conflict of interest: There is no conflict of interest to declare.

Received 24 April 2014; Received in final form 10 May 2014; Accepted 30 May 2014. 
interpretation are relatively simple, does not require specific instruments, and is part of the Unified Parkinson's Disease Rating Scale (UPDRS), routinely used for the rating of PD patients ${ }^{7}$. A detailed description of the PT performance will be described in the Methods section of this study.

The objectives of this study were to evaluate the correlation of PI as measured by the PT scores and the history of falls.

\section{METHOD}

Subjects were recruited among patients followed up at the State of Parana Parkinson Association. This study was approved by the local ethics committee and patients provided informed consent. Standardized data collection included demographics, disease duration, and history of falls.

Inclusion criteria required (i) a diagnosis of PD established by movement disorders experts, based on the Queen Square Brain Bank criteria; (ii) that the patient was not bed or wheelchair bound (H\&Y stage $<5)$; and (iii) was able to come to his/her next follow-up visit in the off medication condition for at least 12 hours. In addition, we did not include cases with a history of other conditions that could interfere with the assessment, including previous cerebrovascular disease, known polyneuropathies, and stereotactic surgery. Disease duration was defined by the approximate time from symptom onset until the moment of data collection. To allow for estimates of time to reach specific landmarks such as disease stage and PI, we matched patients for age of onset. Rating of falls history during the previous 6 months was based on item 13 of the UPDRS part II: 0 - no falls; 1 - rare; 2 - occasional, less than once a day; 3 - on average once a day; 4 - more than once a day ${ }^{7}$. We considered as frequent fallers those with scores $>1$. To avoid for bias in the performance and interpretation of the test, the information regarding the history of falls was only collected after the PT was executed, therefore, during PT performance, the examiner was blinded for fall status.

To minimize differences in the performance of the $\mathrm{PT}$, the test was executed by the same examiner. The PT was performed uniformly according to the following guidelines ${ }^{5,7,9}$ :

- General instructions were provided to each patient, explaining the test and which postural responses would be necessary to regain balance - i.e., adjustment of the truck and/or stepping backward.

- The patient was positioned with his/her back towards the examiner, looking ahead but not bracing forward (tested by placing hands on patient's shoulders). The feet should not be held together, nor kept too far apart, but placed on a comfortable standing position at approximately shoulder width.
- The examiner positioned him/herself at a distance that allowed recovery space but should also be ready to catch the patient. When patients lose PI on the PT, the unstable movement is directed backwards and downwards, either en bloc or with short steps while trying to recover. Therefore, stopping the backward movement may not be enough and the examiner must be ready to hold the falling patient by placing his/her extended arms under the subjects' axillae.

- The tug should be directed to the patients' shoulders (not the proximal arms) with strength capable of displacing the center of gravity from its neutral position while standing, requiring compensatory movements to regain adequate posture. In order to ensure that low scores are not unfairly biased toward larger individuals, the pull is usually considered not strong enough if the subject does not need at least one backward step to regain balance. One gentler advisory push was given before the definitive tug, to avoid the interpretation of a startle response to the unexpected challenge as part of the postural reaction.

The scores of the pull test followed UPDRS recommendations: 0 - normal, i.e. recovers unaided in up to two backward steps; 1 - also recovers unaided but more than two steps are required; 2 - independent of the number of steps, patient would fall if not caught by examiner; 3 - very unstable, may lose balance spontaneously; 4 - unable to stand without assistance ${ }^{7}$.

Statistical analysis was performed as follows: comparison of study findings among different parameters used the two tailed $t$ test for means and the Chi-square test with Yates correction for continuity or Fisher exact test for categorical and ordinal data. Differences were considered significant for $\mathrm{p}<0.05$. Measurements of performance (sensitivity, specificity, positive and negative predictive values) were calculated using the standard formulas. Pearson correlation coefficient was used to measure the strength of the correlation of variables. This correlation ranges from +1 (perfect positive linear relationship between variables) to -1 (perfect negative linear relationship). A coefficient higher than 0.69 indicates a very strong positive relationship.

\section{RESULTS}

A total of 264 patients were included with mean age $67.6 \pm 10$ years (range 40-94), mean age of onset $59.1 \pm 10.7$ years (range 30-87), mean disease duration $8.5 \pm 5$ years (range 1-33), 148 (56.1\%) were male. The scores of the PT showed that $123(46.6 \%)$ had a normal response; 67 (25.4\%) were scored as $1,55(20.8 \%)$ as 2 , and $19(7.2 \%)$ as 3. Due to selection bias inherent in our study's inclusion criteria, no patient was scored as 4 in the PT, as this indicates 
Table 1. Demographic and clinical data of Parkinson's disease cases divided according to pull test score.

\begin{tabular}{lcccccc}
\hline PT score & $\mathrm{n}(\%)$ & Gender $(\%$ male) & Age & Onset & Duration & H\&Y \\
\hline 0 & $123(46.6)$ & 59.3 & 59.7 & $65.6 \pm 8.1$ & $59.1 \pm 8.6$ & $6.5 \pm 3.9$ \\
1 & $67(25.4)$ & 52.7 & $67.2 \pm 11.6$ & $58 \pm 13.4$ & $9.2 \pm 5.2$ & $3 \pm 0.5$ \\
2 & $55(20.8)$ & 47.4 & $70.9 \pm 9.9$ & $59.9 \pm 10.7$ & $11.1 \pm 5$ & $3.5 \pm 0.5$ \\
3 & $19(7.2)$ & $72.4 \pm 10.6$ & $60.6 \pm 12.8$ & $11.7 \pm 5$ & $3.9 \pm 0.2$ \\
\hline
\end{tabular}

PT: pull test; H\&Y: Hoehn \& Yahr scale score.

A

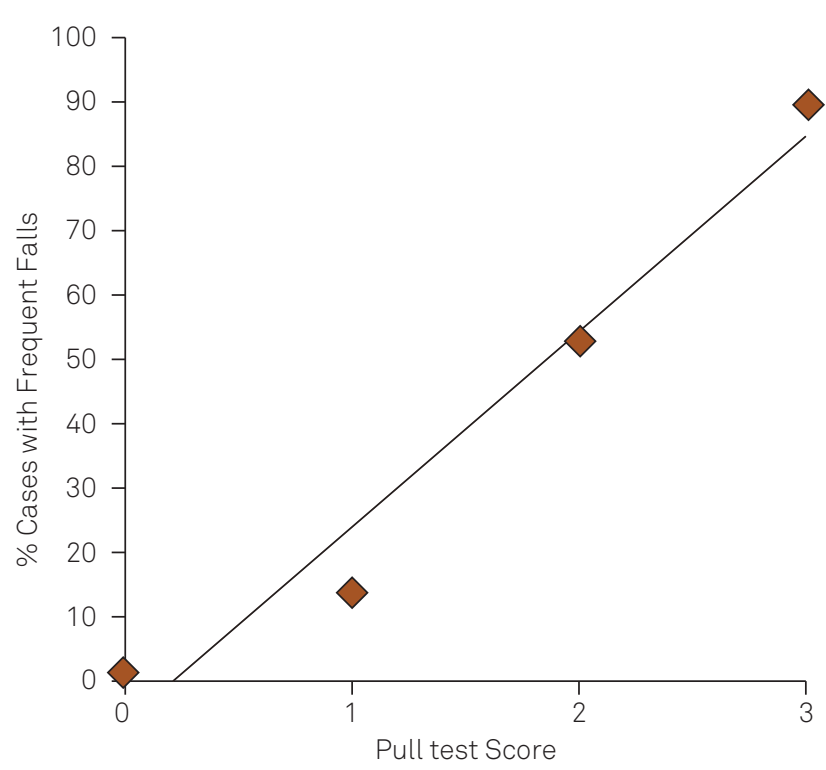

B

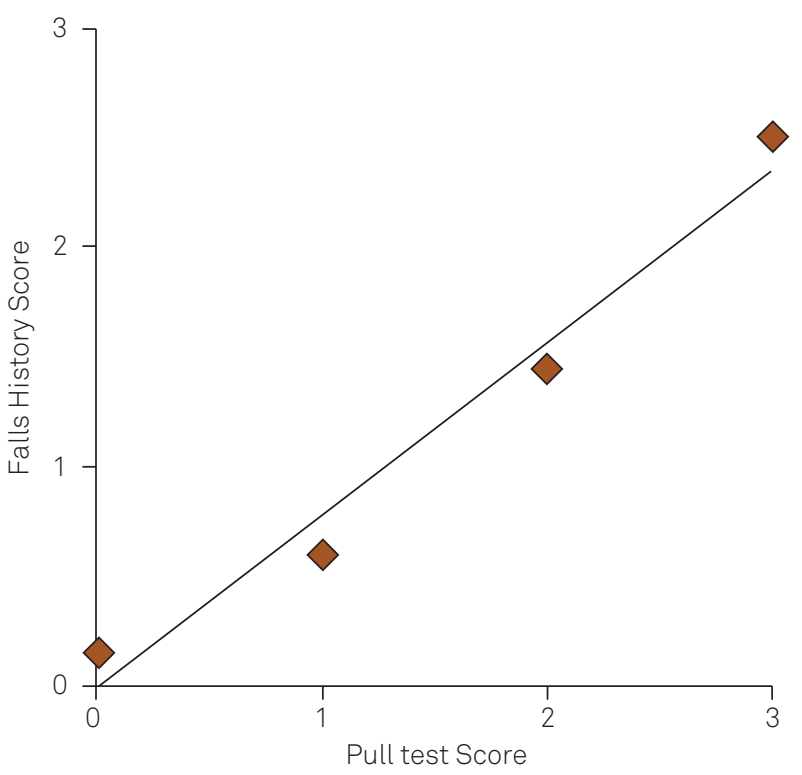

Pearson correlation coefficient: 0.73 .

Figure.. Correlation of the pull test score and proportion of cases with frequent falls (A) and mean falls history score (B).

Table 2. Comparison of pull test score with falls history score and proportion of cases with frequent falls.

\begin{tabular}{|c|c|c|c|c|c|}
\hline PT Score & Falls history & $p$ & Frequent falls & $p$ & OR $(95 \% \mathrm{Cl})$ \\
\hline 0 & $0.15 \pm 0.4$ & $<0.0001$ & $1(0.8 \%)$ & 0.0007 & $18.9(2.3-153)$ \\
\hline 1 & $0.6 \pm 0.7$ & $<0.0001$ & $9(13.4 \%)$ & $<0.0001$ & $7.2(3-17.3)$ \\
\hline 2 & $1.45 \pm 0.8$ & $<0.0001$ & $29(52.7 \%)$ & 0.003 & $9.5(2-45)$ \\
\hline 3 & $2.5 \pm 0.9$ & & $17(89.5 \%)$ & & \\
\hline
\end{tabular}

PT: pull test; OR: odds ration; Cl: confidence interval; p-values represent comparisons with subsequent score, i.e., 0 vs. 1, 1 vs. 2, etc.

patients that are unable to stand, therefore are bed or wheelchair bound, which defines H\&Y stage V. The demographics and other clinical variables for patients with each of these scores are shown in Table 1. The table shows, as expected, that after matching for age of PD onset, worsening of PT scores was correlated with longer disease duration and, obviously, worse severity as measured by the H\&Y scores. Frequency of male patients tended to decrease slightly with higher PT scores, however differences were not significant (p: 0.48 for the two groups with larger difference, PT score 1 vs. 3).

Figure and Table 2 show the comparison and correlation of the PT scores and proportion of cases with frequent falls and mean falls history score. For each increase in the PT scores, both parameters were significantly worse, with a strong positive linear relationship (Pearson correlation coefficient: 0.73). Also, the relative effects of each increase in the score of the PT with the chance of presenting frequent falls (odds ratios) were marked. Finally, the performance of the PT in regards to the history of falls is shown in Table 3. In summary, the table shows that for any abnormal PT score, sensibility and NPV are exceptionally good,

Table 3. Performance of pull test scores for the history of falls.

\begin{tabular}{lcccc} 
PT score & Sensibility (\%) & Specificity (\%) & PPV (\%) & NPV (\%) \\
\hline$>0$ & 98.2 & 59 & 39 & 99.2 \\
$>1$ & 82.1 & 86.5 & 62.2 & 94.7 \\
$>2$ & 30.4 & 99 & 90 & 84.1 \\
\hline PT: pull test; PPV: positive predictive value; NPV: & negative predictive \\
value.
\end{tabular}


while specificity and PPV tend to improve when PT scores increase.

\section{DISCUSSION}

Almost 30 years ago, the PT, with origins in the $19^{\text {th }}$ century with Charcot and Romberg, was incorporated into the UPDRS as a measure of $\mathrm{PI}^{7,10}$. While the test is now not only a part of the routine assessment of patients with parkinsonism, but also an integral part of clinical research, its' performance and interpretation are not uniform. In fact, one of the main criticisms of the PT is that it is wrongly performed and its responses incorrectly interpreted ${ }^{6,9}$.

In our study, we showed that if standardized and established performance guidelines are used, and more rigid and clear interpretation parameters are defined, the clinical data extracted from the test may be clinically useful for the detection of cases at risk for falls or for those in which the history is not clear in this regard. Our findings also showed that for patients with frequent falls, there is a high likelihood that the PT will be abnormal. On the other hand, if the PT is normal there is a high chance that the patient is not a frequent faller. Also, the fact that the PT showed better specificity and PPV only for higher PT scores indicate that the group of PD patients with an abnormal test score of 1 , includes cases with risk of falls who are not yet considered frequent fallers. This group may indeed benefit ideally from prevention and awareness procedures for falls, while those who are already frequent fallers may require more aggressive therapeutic interventions.

A few clinical tests besides the PT have been described for the assessment of PI in PD. The motor part of the UPDRS is widely used to assess motor signs in general, and a subscore of this scale, designated Postural Instability and Gait Disorders (PIGD) subscale, has been used in several studies in the literature ${ }^{11}$. The subscore includes the sum of five items from the UPDRS part II and III: falls (item 13), freezing (item 14), walking (item 15), gait (item 29), and the pull test (item 30). In the One-Leg Stance, subjects are instructed to stand on their preferred leg with hands on their hips and eyes open, looking straight ahead. The outcome is positive when the subject reaches a maximum of 30 seconds without touching the ground or the stance leg with the lifted foot or swinging his/her arms away from the hips ${ }^{12}$. The Functional Reach test is performed with the subject standing with the dominant side next to, but not touching, a wall and then raising the dominant arm to 90 degrees with the hand outstretched. The examiner records the initial position of the fingertips along a meter stick held horizontally against the wall. The subject then tries to reach forward as far as possible without falling, lifting the heels, or moving the feet. The examiner record the difference between the final and initial fingertip positions ${ }^{13}$. The Timed Up and Go (TUG) test has also been used in the geriatric population in general, and previous research has established its reliability and sensitivity to medication states in patients with PD. The test is performed with patients sitting and instructed to stand up when they hear the word "go", walk three meters from the chair, turn around, walk back to the chair and sit as fast as they can, without running. Performance is rated in seconds ${ }^{14}$. The Functional Gait Assessment (FGA) test has been shown to have moderate levels of sensitivity and specificity, comparable to the TUG. The FGA has interesting features, such as the inclusion of items that require sensory integration while dynamic mobility tasks are performed (head turns, walking with eyes closed, and walking backwards) ${ }^{15}$. These features are potentially more specific in predicting falls in persons with PD by exposing the patients to specific ordinary situations. Both the TGU and the FGA have been compared to the PT in regards to falls, showing to be equal or better predictors of falls in $\mathrm{PD}^{16}$. Finally, in the push and release test (PRT), subjects stand in a comfortable stance with eyes open, while the examiner stands behind and instructs subjects to push backward against the palms of the examiners' hands placed on the subjects' scapulae. The examiner must flex his/her elbows to allow backward movement of the trunk while supporting the subjects' weight with their hands. When the subjects' shoulders and hips move to a stable position just behind their heels, the examiner suddenly removes both hands, requiring the subjects to take a backward step to regain balance. The test is scored similarly to the $\mathrm{PT}^{17}$.

Previous studies have tried to assess the efficacy of the PT using other outcome parameters. Bloem et al. ${ }^{18}$, compared the PT with dynamic posturography in patients with PD finding a moderate direct correlation in the off medication state and no correlation in the on state, casting doubt on the use of the clinical test as a measure of PI. More recently, another study analyzed the correlation between the scores of a questionnaire assessing balance confidence during activities of daily living, including the history of falls (designated the $\mathrm{ABC}$ score), and multiple balance tests, such as the UPDRS III scores for gait and PT, the one-leg stance test, the functional reach test, and the PIGD. The authors found that the combination of the UPDRS gait and PT scores, and the one-leg stance test had the best correlation with the $\mathrm{ABC}$ score. The authors conclude that multiple balance tests that assess different types of postural stress provide the optimal assessment of PI in $\mathrm{PD}^{5}$. Another similar study that included multiple tests, including the PT, found that the one-leg stance test, the PT, the functional reach and the tandem stance test are all independently correlated with the risk of falls. The magnitude was predictably superior in regards to identification of subjects with a history of falls when all tests were performed in sequence, in the form 
of a battery ${ }^{19}$. The PRT was also compared with the PT in $\mathrm{PD}$, again in regards to their ability to discriminate fallers and non fallers, showing that both tests presented good accuracy, which was similar when both were compared in the off medication condition. Finally, Visser et al. evaluated the performance and interrater reliability of variants of the PT (expected vs. unexpected pulls; number of backward steps) for the prediction of falls. Their conclusion was that, for trained examiners, interrater reliability and performance were good for all variants. The PT variant with the best performance was the one we intentionally chose to be executed in our study ${ }^{6}$.

Our study has limitations. The patients were not assessed in the "on" condition, therefore the magnitude of the findings may be underestimated. Our study findings also carry the limitations of the PT, including fact that it is supposed to be performed in a standard condition, with one fixed external perturbation (the pull), which does not include the actual circumstances in which falls tend to occur in daily life, such as while turning, getting up from a sitting position, etc. Finally, although we carefully selected our cases in regards to uniformly include subjects with an established final diagnosis of $\mathrm{PD}$, the possibility of inclusion of other forms of degenerative parkinsonism exists. The strengths of our study were the relatively larger number of subjects studied, the facts that all subjects were assessed by the same examiner and the performance of the test, as well as its interpretation, followed rigorous fixed parameters as outlined in the methods section.

In conclusion, accounting for the use of standard parameters for test performance and interpretation, the PT is still seemingly simple, but provides important and reliable information regarding PI and the risk of falls, two of the most disabling problems in moderate to severe stages of $\mathrm{PD}$. The combination of multiple balance tests is probably advantageous and encouraged.

\section{Acknowledgements}

Dr. Anthony E. Lang from the Movement Disorders Centre, University of Toronto, contributed and critically reviewed the description of the PT performance in the Methods section of this manuscript.

\section{References}

1. Pickering RM, Grimbergen YA, Rigney $U$, et al. A meta-analysis of six prospective studies of falling in Parkinson's disease. Mov Disord 2007;22:1892-1900.

2. Hoehn MM, Yahr MD. Parkinsonism: onset, progression and mortality. Neurology 1967;17:427-442.

3. Munhoz RP, Werneck LC, Teive HA. The differential diagnoses of parkinsonism: findings from a cohort of 1528 patients and a 10 years comparison in tertiary movement disorders clinics. Clin Neurol Neurosurg 2010;112:431-435.

4. Adkin AL, Frank JS, Jog MS. Fear of falling and postural control in Parkinson's disease. Mov Disord 2003;18:496-502.

5. Jacobs JV, Horak FB, Tran VK, Nutt JG. Multiple balance tests improve the assessment of postural stability in subjects with Parkinson's disease. J Neurol Neurosurg Psychiatry 2006;77:322-326.

6. Visser M, Marinus J, Bloem BR, Kisjes H, van den Berg BM, van Hilten $\mathrm{JJ}$. Clinical tests for the evaluation of postural instability in patients with parkinson's disease. Arch Phys Med Rehabil 2003;84:1669-1674.

7. Goetz CG, Tilley BC, Shaftman SR, et al; Movement Disorder Society UPDRS Revision Task Force. Movement Disorder Society-sponsored revision of the Unified Parkinson's Disease Rating Scale (MDSUPDRS): scale presentation and clinimetric testing results. Mov Disord 2008;23:2129-2170.

8. Hughes AJ, Daniel SE, Kilford L, Lees AJ. Accuracy of clinical diagnosis of idiopathic Parkinson's disease: a clinico-pathological study of 100 cases. J Neurol Neurosurg Psychiatry 1992;55:181-184.

9. Munhoz RP, Li J-Y, Kurtinecz MA, et al. Evaluation of pull test technique in assessing postural instability in Parkinson's disease. Neurology 2004;62:125-127.
10. Hunt AL, Sethi KD. The pull test: a history. Mov Disord 2006;21:894-899.

11. Lozano AM, Lang AE, Galvez-Jimenez N, et al. Effect of GPi pallidotomy on motor function in Parkinson's disease. Lancet 1995;346:1383-1387.

12. Anemaet W, Moffa-Trotter M. Functional tools for assessing balance and gait impairments. Topics Geriatric Rehab 1999;15:66-83.

13. Weiner DK, Duncan PW, Chandler J, Studenski SA. Functional reach a marker of physical frailty. J Am Geriatr Soc 1992;40:203-207.

14. Morris S, Morris ME, lansek R. Reliability of measurements obtained with the Timed "Up \& Go" test in people with Parkinson disease. Phys Ther 2001;81:810-818.

15. Wrisley DM, Marchetti GF, Kuharsky DK, Whitney SL. Reliability, internal consistency, and validity of data obtained with the functional gait assessment. Phys Ther 2004;84:906-918.

16. Foreman KB, Addison O, Kim HS, Dibble LE. Testing balance and fall risk in persons with Parkinson disease, an argument for ecologically valid testing. Parkinsonism Relat Disord 2011;17:166-171.

17. Valkovic P, Brozová H, Bötzel K, Růzicka E, Benetin J. Push-andrelease test predicts Parkinson fallers and nonfallers better than the pull test: comparison in OFF and ON medication states. Mov Disord 2008;23:1453-1457.

18. Bloem BR, Beckley DJ, van Hilten BJ, Roos RA. Clinimetrics of postural instability in Parkinson's disease. J Neurol 1998;245:669-673.

19. Smithson F, Morris ME, lansek R. Performance on clinical tests of balance in Parkinson's disease. Phys Ther 1998;78:577-592. 